\title{
CONTINUOUS DEPENDENCE AND DIFFERENTIATION OF SOLUTIONS OF FINITE DIFFERENCE EUQATIONS
}

\section{JOHNNY HENDERSON and LINDA LEE}

Department of Algebra, Combinatorics and Analysis

Auburn University

Auburn, Alabama 36849 U.S.A.

(Received August 10, 1990 and in revised form March 9, 1991)

\section{Abstract}

Conditions are given for the continuity and differentiability of solutions of initial value problems and boundary value problems for the $n^{\text {th }}$ order finite difference equation, $u(m+n)=$ $f(m, u(m), u(m+1), \ldots, u(m+n-1)), m \in \mathbf{Z}$.

KEYWORDS AND PHRASES. Finite difference equation, initial value problem, boundary value problem.

1980 AMS CLASSIFICATION CODE. 39A10, 39A12, 34B10.

\section{Introduction}

Let $\mathbf{Z}$ denote the integers, and given $a<b$ in $\mathbf{Z}$, let $[a, \infty)=\{a, a+1, \ldots\},[a, b]=\{a, a+$ $1, \ldots, b\},[a, b)=\{a, \ldots, b-1\}$, with $(a, \infty),(a, b)$, etc., being similarly defined. In this paper, we will be concerned with solutions of the $n^{\text {th }}$ order difference equation,

$$
u(m+n)=f(m, u(m), \ldots, u(m+n-1))
$$

where

(A) $f\left(m, u_{1}, \ldots, u_{n}\right): \mathbf{Z} \times \mathbf{R}^{n} \rightarrow \mathbf{R}$ is continuous.

We will also assume in many settings the condition:

(B) $\frac{\partial f}{\partial u_{1}}\left(m, u_{1}, \ldots, u_{n}\right): \mathbf{Z} \times \mathbf{R}^{n} \rightarrow \mathbf{R}$ are continuous, for $1 \leq i \leq n$.

We will present results about continuous dependence and differentiation of solutions of (1.1) with respect to initial values and certain boundary values. Also, given a solution $u(m)$ of $(1.1)$, we will be interested in solutions of the linear equation,

$$
z(m+n)=\sum_{i=1}^{n} \frac{\partial f}{\partial u_{i}}(m, u(m), \ldots, u(m+n-1)) z(m+i-1) .
$$

Equation (1.2) is called the variational equation along the solution $u(m)$ of (1.1).

Many studies have been devoted to finite difference equations. Following Hartman's [1] major paper, a number of recent papers have appeared that are devoted to boundary value problems for finite difference equations. For example, papers by Ahlbrandt and Hooker [2], Eloe [3], [4], 
Hankerson [5], [6], Hankerson and Peterson [7], Hooker and Patula [8], Ladas et al. [9], Peterson [10] - [12], and Smith and Taylor [13] have dealt with disconjugacy or oscillation and nonoscillation of linear difference equations, while the works by Eloe [14] - [16], Peil [17], and Peterson [12] have also dealt with disfocality criteria for linear difference equations. For the nonlinear equation, (1.1), Agarwal [18], Eloe [3], [4], Hankerson [5], [6], Henderson [19] - [21], and Peterson [12] have addressed questions concerning boundary value problems.

As in several of the above cited works, the results obtained in this paper are motivated as analogues of results from ordinary differential equations. In the case of initial value problems for ordinary differential equations, Hartman [22] presents a theorem due to Peano in which solutions are differentiated with respect to initial conditions. Subsequent to that, for boundary value problems associated with ordinary differential equations, several authors have obtained results concerning differentiation of solutions with respect to boundary conditions; e.g., see Peterson [10] - [12] and Henderson [23], [24]. In fact, Brantley and Henderson [25] and Henderson [24] have given some fairly complete analogues of the Peano theorem for certain boundary value problems for nonlinear ordinary differential equations.

The primary motivation for this paper arises from the studies by Hankerson [5] and Peterson [12], which are devoted to differentiation of solutions of finite difference equations with respect to boundary values for "two-point" boundary value problems.

In Section 2, we state two results for solutions of initial value problems for (1.1). The first result establishes that under condition (A), solutions of initial value problems for (1.1) depend continuously on initial values. The second result states an analogue of the Peano theorem, in that, under conditions (A) and (B), solutions of initial value problems for (1.1) can be differentiated with respect to initial values.

In Section 3, we present the main theorems of the paper. We establish analogues of our results obtained in Section 2, for conjugate boundary value problems for (1.1). The proofs in this section depend on uniqueness of solutions of conjugate boundary value problems. We will assume at the appropriate places that we have this uniqueness.

\section{Differentiation of Solutions with Respect to Initial Values}

The $n^{\text {th }}$ order difference equation (1.1) in conjunction with the conditions

$$
u\left(m_{0}+i-1\right)=u_{i}, 1 \leq i \leq n,
$$

where $m_{0} \in \mathbf{Z}, u_{i} \in \mathbf{R}, 1 \leq i \leq n$, is called an initial value problem. In this section, we state two theorems regarding continuous dependence and differentiability with respect to initial values for solutions of (1.1), (2.1). All that is required for the proofs of these theorems is a conversion of (1.1), (2.1) to an initial value problem for a first order system, $U(m+1)=F(m, U(m)), U\left(m_{0}\right)=U_{0}$, and then provide obvious modifications of proofs in Hartman [22]. For this reason, we will omit their proofs. However, these two theorems will play a fundamental role in the results of the next section. We also remark that solutions of initial value problems, $(1.1),(2.1)$, are unique on $\left[m_{0}, \infty\right), m_{0} \in \mathbf{Z}$. 
THEOREM 2.1. (Continuous dependence on initial values) Suppose (A) is satisfied. Let $u\left(m ; m_{0}, u_{1}, u_{2}, \ldots, u_{n}\right)$ be the solution of $(1.1),(2.1)$ on $\left[m_{0},+\infty\right)$, where $m_{0} \in \mathbf{Z}, u_{1}, u_{2}, \ldots, u_{n} \in$ R. Then, given $\epsilon>0, k \in \mathbf{N}$, there exists a $\delta\left(\epsilon, m_{0}, k, u_{1}, u_{2}, \ldots, u_{n}\right)>0$, such that if $\left|u_{i}-v_{\imath}\right|<$ $\delta, 1 \leq i \leq n$, then $\left|u\left(m ; m_{0}, u_{1}, u_{2}, \ldots, u_{n}\right)-u\left(m ; m_{0}, v_{1}, v_{2}, \ldots, v_{n}\right)\right|<\epsilon$, for every $m \in\left[m_{0}, m_{0}+\right.$ $k]$.

We now state our analogue of Peano's theorem in Hartman [22] for the scalar initial value problem (1.1), (2.1).

THEOREM 2.2. Assume $f$ satisfies (A) and (B). Let $m_{0} \in \mathbf{Z}$, and $u_{1}, u_{2}, \ldots, u_{n} \in \mathbf{R}$ be given. If $u(m)=u\left(m ; m_{0}, u_{1}, \ldots, u_{n}\right)$ denotes the solution of the initial value problem, (1.1), (2.1), then, for every $1 \leq j \leq n$, given $v_{1}, \ldots, v_{n} \in \mathbf{R}$,

$$
\beta_{j}(m)=\frac{\partial u}{\partial u_{j}}\left(m ; m_{0}, v_{1}, \ldots, v_{n}\right)
$$

exists and is the solution of the variational equation,

$$
\begin{aligned}
\beta_{3}(m+n)= & \sum_{i=1}^{n} \frac{\partial f}{\partial x_{i}}\left(m, u\left(m ; m_{0}, v_{1}, \ldots, v_{n}\right), u\left(m+1 ; m_{0}, v_{1}, \ldots, v_{n}\right), \ldots,\right. \\
& \left.u\left(m+n-1 ; m_{0}, v_{1}, \ldots, v_{n}\right)\right) \beta_{j}(m+i-1),
\end{aligned}
$$

and satisfies

$$
\beta_{j}\left(m_{0}+i-1\right)=\delta_{i j}, 1 \leq i \leq n
$$

\section{Differentiation of Solutions with Respect to Boundary Values}

In this section, we prove analogues of Theorems 2.1 and 2.2 for conjugate boundary value problems for (1.1).

DEFINITION 3.1. [21] Given $m_{1}<\ldots<m_{n}$ in $\mathbf{Z}$ and $u_{1}, \ldots, u_{n} \in \mathbf{R}$, a boundary value problem for (1.1) satisfying

$$
u\left(m_{i}\right)=u_{i}, 1 \leq i \leq n
$$

is called a conjugate boundary value problem.

Conjugate boundary value problems for (1.1) (both nonlinear and linear cases) have received much recent attention. Much of this attention has arisen from the paper by Hartman [1] in which he gave conditions characterizing disconjugacy for linear difference equations in terms of generalized zeros.

DEFINITION 3.2. [1] Let $u: \mathbf{Z} \rightarrow \mathbf{R}$. We say that $u$ has a generalized zero at $m_{0}$ provided, either $u\left(m_{0}\right)=0$ or there is a $k \in \mathbf{N}$ such that $(-1)^{k} u\left(m_{0}-k\right) u\left(m_{0}\right)>0$ and if $k>1, u\left(m_{0}-k+1\right)=$ $\ldots=u\left(m_{0}-1\right)=0$.

DEFINITION 3.3. [21] The nonlinear difference equation, (1.1), is said to be disconjugate on $\mathbf{Z}$ provided that whenever $u(m)$ and $v(m)$ are solutions of $(1.1)$ such that $u(m)-v(m)$ has $\mathrm{n}$ generalized zeroes at $m_{1}<m_{2}<\ldots<m_{n} \in \mathbf{Z}$, it follows that $u(m)-v(m) \equiv 0$ on $\left[m_{1}, \infty\right)$. 
To be complete, we formulate Definition 3.3 as Hartman did for the case when (1.1) is linear, say, for the equation,

$$
v(m+n)=\sum_{i=1}^{n} \alpha_{i}(m) v(m+i-1),
$$

where $\alpha_{i}: \mathbf{Z} \rightarrow \mathbf{R}, 1 \leq i \leq n$.

DEFINITION 3.4. [1] The linear equation, (3.2), is said to be disconjugate on $\mathbf{Z}$ provided there is no nontrivial solution $u$ of equation (3.2) which has $n$ generalized zeros on $\mathbf{Z}$.

For the remainder of this chapter, we adopt the following notation to distinguish boundary value problems from initial value problems. For $m_{1}<\ldots<m_{n}$ in $\mathbf{Z}$ and $u_{1}, \ldots, u_{n} \in \mathbf{R}$, let $u(m)=u\left(m ; m_{1}, \ldots, m_{n}, u_{1}, \ldots, u_{n}\right)$ denote the solution of the boundary value problem, (1.1), (3.1). And, for $m_{0} \in \mathbf{Z}$ and $c_{1}, \ldots, c_{n} \in \mathbf{R}$, we will let $v(m)=v\left(m ; m_{0}, c_{1}, \ldots, c_{n}\right)$ denote the solution of the initial value problem, (1.1), (2.1), (i.e., $\left.v\left(m_{0}+i-1\right)=c_{i}, 1 \leq i \leq n\right)$.

The results of this section are patterned somewhat after those in Hankerson [5]. Following that route, we obtain analogues of Theorems 2.1 and 2.2 for conjugate boundary value problems (1.1), (3.1). We will have need of disconjugacy assumptions on (1.1) and on the variational equation, (1.2). Our results on continuous dependence make use of the Brouwer Theorem on Invariance of Domain which we state here for convenience.

THEOREM 3.5. If $U$ is an open subset of $\mathbf{R}^{n}, n$ dimensional Euclidean space, and $\phi: U \rightarrow$ $\mathbf{R}^{n}$ is one to one and continuous on $U$, then $\phi$ is a homeomorphism and $\phi(U)$ is an open subset of $\mathbf{R}^{n}$.

THEOREM 3.6. (Continuous dependence on boundary values) Suppose condition (A) is satisfied and that (1.1) is disconjugate on $\mathbf{Z}$. Let $y(m)$ be a solution of $(1.1)$ on $\left[m_{1}, \infty\right)$, and let $m_{1}<m_{2}<\ldots<m_{n}$ in $\left[m_{1}, \infty\right)$ be given. Then, there exists an $\epsilon>0$ such that, if $\gamma_{1}, \gamma_{2}, \ldots, \gamma_{n} \in \mathbf{R}$, with $\left|\gamma_{i}\right|<\epsilon, i=1,2, \ldots, n$, then the boundary value problem for (1.1) satisfying

$$
u\left(m_{i}\right)=y\left(m_{i}\right)+\gamma_{i}, 1 \leq i \leq n
$$

has a unique solution $u\left(m ; m_{1}, \ldots, m_{n}, y\left(m_{1}\right)+\gamma_{1}, \ldots, y\left(m_{n}\right)+\gamma_{n}\right)$. Furthermore, as $\epsilon \rightarrow 0$, the solutions $u\left(m ; m_{1}, \ldots, m_{n}, y\left(m_{1}\right)+\gamma_{1}, \ldots, y\left(m_{n}\right)+\gamma_{n}\right)$ converge to $y(m)$ on $\left[m_{1}, \infty\right)$.

PROOF. Let $m_{1}<m_{2}<\ldots<m_{n} \in\left[m_{1}, \infty\right)$. Define $\phi: \mathbf{R}^{n} \rightarrow \mathbf{R}^{n}$ by $\phi\left(c_{1}, c_{2}, \ldots, c_{n}\right)=$ $\left(v\left(m_{1}\right), v\left(m_{2}\right), \ldots, v\left(m_{n}\right)\right)$, where, as from above, $v(m)=v\left(m ; m_{1}, c_{1}, \ldots, c_{n}\right)$ is the solution of the initial value problem for (1.1) satisfying

$$
v\left(m_{1}+i-1\right)=c_{i}, 1 \leq i \leq n
$$

We claim that $\phi$ is one to one.

Suppose $\phi\left(c_{1}, \ldots, c_{n}\right)=\phi\left(c_{1}^{\prime}, \ldots, c_{n}^{\prime}\right)$. Then,

$$
\begin{aligned}
& \left(v\left(m_{1} ; m_{1}, c_{1}, \ldots, c_{n}\right), v\left(m_{2} ; m_{1}, c_{1}, \ldots, c_{n}\right), \ldots, v\left(m_{n} ; m_{1}, c_{1}, \ldots, c_{n}\right)\right) \\
& \quad=\left(v\left(m_{1} ; m_{1}, c_{1}^{\prime}, \ldots, c_{n}^{\prime}\right), v\left(m_{2} ; m_{1}, c_{1}^{\prime}, \ldots, c_{n}^{\prime}\right), \ldots, v\left(m_{n} ; m_{1}, c_{1}^{\prime}, \ldots, c_{n}^{\prime}\right)\right)
\end{aligned}
$$

By the assumed disconjugacy of (1.1) on $\mathbf{Z}$, (in particular, by uniqueness of solutions to (1.1), (3.1)), 


$$
v\left(m ; m_{1}, c_{1}, \ldots, c_{n}\right)=v\left(m ; m_{1}, c_{1}^{\prime}, \ldots, c_{n}^{\prime}\right), \text { for every } m \in\left[m_{1},+\infty\right)
$$

As a consequence,

$$
v\left(m_{1}+i-1 ; m_{1}, c_{1}, \ldots, c_{n}\right)=v\left(m_{1}+i-1 ; m_{1}, c_{1}^{\prime}, \ldots, c_{n}^{\prime}\right), 1 \leq i \leq n .
$$

In particular, $\left(c_{1}, \ldots, c_{n}\right)=\left(c_{1}^{\prime}, \ldots, c_{n}^{\prime}\right)$. Hence, $\phi$ is one-to-one.

Now, we claim that $\phi$ is continuous. Suppose $\left\langle\left(c_{1}^{l}, c_{2}^{l}, \ldots, c_{n}^{l}\right)\right\rangle$ converges to $\left(c_{1}, c_{2}, \ldots, c_{n}\right)$ as $\ell \rightarrow \infty$. By continuous dependence on initial conditions, given by Theorem $2.1, v\left(m ; m_{1}, c_{1}^{\ell}, \ldots, c_{n}^{\ell}\right)$ converges to $v\left(m ; m_{1}, c_{1}, \ldots, c_{n}\right)$, for every $m \in\left[m_{1},+\infty\right)$, as $\ell \rightarrow \infty$. In particular,

$$
\lim _{\ell \rightarrow \infty} v\left(m_{i} ; m_{1}, c_{1}^{\ell}, \ldots, c_{n}^{\ell}\right)=v\left(m_{i} ; m_{1}, c_{1}, \ldots, c_{n}\right), 1 \leq i \leq n
$$

Therefore, $\left\langle\phi\left(c_{1}^{\ell}, \ldots, c_{n}^{\ell}\right)\right\rangle$ converges to $\phi\left(c_{1}, \ldots, c_{n}\right)$ as $\ell \rightarrow \infty$, and $\phi$ is continuous.

By the Brouwer Theorem on Invariance of Domain, $\phi$ is a homeomorphism onto the range, $\phi\left(\mathbf{R}^{n}\right)$, and $\phi\left(\mathbf{R}^{n}\right)$ is open in $\mathbf{R}^{n}$. Now, with $y(m)$ the solution in the statement of the Theorem, $\left(y\left(m_{1}\right), y\left(m_{2}\right), \ldots, y\left(m_{n}\right)\right) \in \phi\left(\mathbf{R}^{n}\right)$. Since $\phi\left(\mathbf{R}^{n}\right)$ is open, there exists an $\epsilon>0$, such that if $\left|\gamma_{i}\right|<\epsilon, i=1,2, \ldots, n$, then $\left(y\left(m_{1}\right)+\gamma_{1}, y\left(m_{2}\right)+\gamma_{2}, \ldots, y\left(m_{n}\right)+\gamma_{n}\right) \in \phi\left(\mathbf{R}^{n}\right)$. Now, there exists a unique $\left(r_{1}, \ldots, r_{n}\right)$ such that

$$
\phi\left(r_{1}, \ldots, r_{n}\right)=\left(y\left(m_{1}\right)+\gamma_{1}, y\left(m_{2}\right)+\gamma_{2}, \ldots, y\left(m_{n}\right)+\gamma_{n}\right)
$$

But by definition,

$$
\phi\left(r_{1}, \ldots, r_{n}\right)=\left(v\left(m_{1} ; m_{1}, r_{1}, \ldots, r_{n}\right), v\left(m_{2} ; m_{1}, r_{1}, \ldots, r_{n}\right), \ldots, v\left(m_{n} ; m_{1}, r_{1}, \ldots, r_{n}\right)\right)
$$

where $v\left(m ; m_{1}, r_{1}, \ldots, r_{n}\right)$ is the solution of the initial value problem for (1.1), satisfying

$$
v\left(m_{1}+i-1\right)=r_{i}, 1 \leq i \leq n
$$

Hence,

$$
\begin{aligned}
& \left(y\left(m_{1}\right)+\gamma_{1}, y\left(m_{2}\right)+\gamma_{2}, \ldots, y\left(m_{n}\right)+\gamma_{n}\right) \\
& \quad=\left(v\left(m_{1} ; m_{1}, r_{1}, \ldots, r_{n}\right), v\left(m_{2} ; m_{1}, r_{1}, \ldots, r_{n}\right), \ldots, v\left(m_{n} ; m_{1}, \dot{r}_{1}, \ldots, r_{n}\right)\right)
\end{aligned}
$$

i.e., $v\left(m ; m_{1}, r_{1}, \ldots, r_{n}\right)$ is the solution of $(1.1)$ satisfying

$$
v\left(m_{i} ; m_{1}, r_{1}, \ldots, r_{n}\right)=y\left(m_{i}\right)+\gamma_{i}, 1 \leq i \leq n
$$

For each $j \in \mathbf{N}$, consider

$$
\left(y\left(m_{1}\right)+\gamma_{1}^{j}, y\left(m_{2}\right)+\gamma_{2}^{j}, \ldots, y\left(m_{n}\right)+\gamma_{n}^{j}\right) \in \phi\left(\mathbf{R}^{n}\right)
$$

where

$$
\left|\gamma_{k}^{j}\right|<\epsilon, 1 \leq k \leq n,
$$

and

$$
\lim _{j \rightarrow \infty}\left(y\left(m_{1}\right)+\gamma_{1}^{j}, \ldots, y\left(m_{n}\right)+\gamma_{n}^{\jmath}\right)=\left(y\left(m_{1}\right), \ldots, y\left(m_{n}\right)\right) .
$$

For each $j \in \mathbf{N}$, let 


$$
u_{j}(m)=u\left(m ; m_{1}, \ldots, m_{n}, y\left(m_{1}\right)+\gamma_{1}^{\jmath}, \ldots, y\left(m_{n}\right)+\gamma_{n}^{\jmath}\right) .
$$

Now, $\phi^{-1}: \phi\left(\mathbf{R}^{n}\right) \rightarrow \mathbf{R}^{n}$ is continuous. Therefore,

$$
\begin{aligned}
\lim _{j} & \varphi^{-1}\left(u_{j}\left(m_{1}\right), u_{\jmath}\left(m_{2}\right), \ldots, u_{\jmath}\left(m_{n}\right)\right) \\
& =\lim _{i \rightarrow \infty} \phi^{-1}\left(y\left(m_{1}\right)+\gamma_{1}^{j}, y\left(m_{2}\right)+\gamma_{2}^{j}, \ldots, y\left(m_{n}\right)+\gamma_{n}^{j}\right) \\
& =\phi^{-1}\left(\lim _{i \rightarrow \infty}\left(y\left(m_{1}\right)+\gamma_{1}^{j}, y\left(m_{2}\right)+\gamma_{2}^{j}, \ldots, y\left(m_{n}\right)+\gamma_{n}^{j}\right)\right) \\
& =\phi^{-1}\left(y\left(m_{1}\right), y\left(m_{2}\right), \ldots, y\left(m_{n}\right)\right)
\end{aligned}
$$

i.e., the initial values of $u_{i}(m)$ converge to the initial values of $y(m)$. By Theorem 2.1, $u_{i}(m)$ converges uniformly to $y(m)$ on each compact subset of $\left[m_{1}, \infty\right)$. It follows that the solutions in the statement of the Theorem, $u\left(m ; m_{1}, \ldots, m_{n}, y\left(m_{1}\right)+\gamma_{1}, \ldots, y\left(m_{n}\right)+\gamma_{n}\right)$, converge to $y(m)$, as $\epsilon \rightarrow 0$. The proof is complete.

We now establish our analogue of Theorem 2.2 for conjugate problems.

THEOREM 3.7. (Differentiation of solutions of (1.1) with respect to boundary values) Assume that $f$ satisfies $(A)$ and $(B)$, that (1.1) is disconjugate on $\mathbf{Z}$, and that the variational equation (1.2) is disconjugate along all solutions of (1.1). Let $u(m)=u\left(m ; m_{1}, \ldots, m_{n}, u_{1}, \ldots, u_{n}\right)$ be the solution of (1.1), (3.1) on $\left[m_{1}, \infty\right)$. Then, for $1 \leq j \leq n, \frac{\partial u}{\partial u}$, exists on $\left[m_{1}, \infty\right)$, and $z_{j}(m) \equiv \frac{\partial u}{\partial u}(m)$ is the solution of the variational equation, (1.2), along $u(m)$ and satisfies,

$$
z_{j}\left(m_{i}\right)=\delta_{i j}, 1 \leq i \leq n
$$

PROOF. We consider $\frac{\partial u}{\partial u}$, for fixed $j, 1 \leq j \leq n$. Let $\epsilon>0$ be as in Theorem 3.6. Let $0<|h|<\epsilon$ be given and consider the quotient,

$$
\begin{aligned}
z_{j h}(m)= & \frac{1}{h}\left[u\left(m ; m_{1}, \ldots, m_{n}, u_{1}, u_{2}, \ldots, u_{j}+h, \ldots, u_{n}\right)\right. \\
& \left.-u\left(m ; m_{1}, \ldots, m_{n}, u_{1}, \ldots, u_{j}, \ldots, u_{n}\right)\right] .
\end{aligned}
$$

It suffices to show that $\lim _{h \rightarrow 0} z_{j h}(m)$ exists on $\left[m_{1},+\infty\right)$.

Observe first that,

$$
z_{j h}\left(m_{i}\right)=\delta_{i j}, 1 \leq i \leq n,
$$

for every $h \neq 0$.

For $2 \leq i \leq n$, let $\alpha_{i}=u\left(m_{j}+i-1 ; m_{1}, \ldots, m_{n}, u_{1}, \ldots, u_{n}\right)$, and $\epsilon_{i}=\epsilon_{i}(h)=u\left(m_{j}+\right.$ $\left.i-1 ; m_{1}, \ldots, m_{n}, u_{1}, \ldots, u_{j}+h, \ldots, u_{n}\right)-\alpha_{i}$. Note that $u\left(m_{j} ; m_{1}, \ldots, m_{n}, u_{1}, \ldots, u_{n}\right)=u_{j}$ and $u\left(m_{j} ; m_{1}, \ldots, m_{n}, u_{1}, \ldots, u_{j}+h, \ldots, u_{n}\right)=u,+h$. By Theorem 3.6, $\epsilon_{\imath}(h)$ converges to zero, as $h$ converges to zero, for $2 \leq i \leq n$.

Recalling our notation, $v\left(m ; m_{0}, v_{1}, \ldots, v_{n}\right)$ denotes the solution of the initial value problem for (1.1), satisfying

$$
v\left(m_{0}+i-1\right)=v_{i}, 1 \leq i \leq n .
$$

Then, by using a telescoping sum,

$$
z_{j h}(m)=\frac{1}{h}\left[u\left(m ; m_{1}, \ldots, m_{n}, u_{1}, \ldots, u_{j}+h, \ldots, u_{n}\right)-u\left(m ; m_{1}, \ldots, m_{n}, u_{1}, \ldots, u_{n}\right)\right]
$$




$$
\begin{aligned}
= & \frac{1}{h}\left[v\left(m ; m_{\jmath}, u_{\jmath}+h, \alpha_{2}+\epsilon_{2}, \ldots, \alpha_{n}+\epsilon_{n}\right)-v\left(m ; m_{\jmath}, u_{\jmath}, \alpha_{2}, \ldots, \alpha_{n}\right)\right] \\
= & \frac{1}{h}\left[\left[v\left(m ; m_{\jmath}, u_{\jmath}+h, \alpha_{2}+\epsilon_{2}, \ldots, \alpha_{n}+\epsilon_{n}\right)-v\left(m ; m_{\jmath}, u_{\jmath}, \alpha_{2}+\epsilon_{2}, \ldots, \alpha_{n}+\epsilon_{n}\right)\right]\right. \\
& +\left[v\left(m ; m_{\jmath}, u_{\jmath}, \alpha_{2}+\epsilon_{2}, \ldots, \alpha_{n}+\epsilon_{n}\right)-v\left(m ; m_{j}, u_{\jmath}, \alpha_{2}, \alpha_{3}+\epsilon_{3}, \ldots, \alpha_{n}+\epsilon_{n}\right)\right] \\
& \left.+\ldots+\left[v\left(m ; m_{\jmath}, u_{j}, \alpha_{2}, \ldots, \alpha_{n-1}, \alpha_{n}+\epsilon_{n}\right)-v\left(m ; m_{\jmath}, u_{j}, \alpha_{2}, \ldots, \alpha_{n-1}, \alpha_{n}\right)\right]\right] .
\end{aligned}
$$

Now, by Theorem 2.2, solutions of (1.1) can be differentiated with respect to initial values; i.e., $\beta_{1}=\frac{\partial v}{\partial v_{1}}, \beta_{2}=\frac{\partial v}{\partial v_{2}}, \ldots, \beta_{3}=\frac{\partial v}{\partial v_{j}}, \ldots, \beta_{n}=\frac{\partial v}{\partial v_{n}}$ exist. Hence, by Theorem 2.2 and the Mean Value Theorem, we have that

$$
\begin{aligned}
z_{j h}(m)= & \frac{1}{h}\left[\beta_{1}\left(m ; v\left(m ; m_{\jmath}, u_{\jmath}+\bar{h}, \alpha_{2}+\epsilon_{2}, \ldots, \alpha_{n}+\epsilon_{n}\right)\right) h\right. \\
& +\beta_{2}\left(m ; v\left(m ; m_{j}, u_{\jmath}, \alpha_{2}+\bar{\epsilon}_{2}, \ldots, \alpha_{n}+\epsilon_{n}\right)\right) \epsilon_{2} \\
& \left.+\ldots+\beta_{n}\left(m ; v\left(m ; m_{\jmath}, u_{\jmath}, \alpha_{2}, \ldots, \alpha_{n-1}, \alpha_{n}+\bar{\epsilon}_{n}\right)\right) \epsilon_{n}\right],
\end{aligned}
$$

where,

$$
\begin{aligned}
& \beta_{1}\left(m ; v\left(m ; m_{\jmath}, u_{j}+\bar{h}, \alpha_{2}+\epsilon_{2}, \ldots, \alpha_{n}+\epsilon_{n}\right)\right)=\frac{\partial v}{\partial v_{1}}\left(m ; m_{j} ; u_{j}+\bar{h}, \alpha_{2}+\epsilon_{2}, \ldots, \alpha_{n}+\epsilon_{n}\right), \\
& \vdots \\
& \beta_{n}\left(m ; v\left(m ; m_{\jmath}, u_{j}, \alpha_{2}, \ldots, \alpha_{n-1}, \alpha_{n}+\bar{\epsilon}_{n}\right)\right)=\frac{\partial v}{\partial v_{n}}\left(m ; m_{j}, u_{j}, \alpha_{2}, \ldots, \alpha_{n}+\bar{\epsilon}_{n}\right),
\end{aligned}
$$

and where $\bar{h}$ is between 0 and $h$, and $\overline{\epsilon_{i}}$ is between 0 and $\epsilon_{i}$, for $i=2, \ldots, n$. That is, $\beta_{1}\left(m ; v\left(m ; m_{j}, u\right.\right.$, $\left.\left.+\bar{h}, \alpha_{2}+\epsilon_{2}, \ldots, \alpha_{n}+\epsilon_{n}\right)\right)$ denotes the solution of the variational equation, (1.2), along $v\left(m ; m_{j}, u_{j}+\right.$ $\left.\bar{h}, \alpha_{2}+\epsilon_{2}, \ldots, \alpha_{n}+\epsilon_{n}\right)$ and satisfies, $\beta_{1}\left(m_{j}+i-1\right)=\delta_{i 1}, 1 \leq i \leq n$. Similarly, $\beta_{2}\left(m ; v\left(m ; m_{j}, u_{j}, \alpha_{2}+\right.\right.$ $\left.\left.\bar{\epsilon}_{2}, \ldots, \alpha_{n}+\epsilon_{n}\right)\right)$ denotes the solution of the variational equation, (1.2), along $v\left(m ; m_{\jmath}, u_{\jmath}, \alpha_{2}+\right.$ $\left.\bar{\epsilon}_{2}, \ldots, \alpha_{n}+\epsilon_{n}\right)$, and satisfies, $\beta_{2}(m,+i-1)=\delta_{\imath 2}, 1 \leq i \leq n$. Continuing, $\beta_{n}\left(m ; v\left(m ; m_{j}, u_{j}, \alpha_{2}, \ldots\right.\right.$, $\left.\alpha_{n-1}, \alpha_{n}+\bar{\epsilon}_{n}\right)$ ) denotes the solution of the variational equation, (1.2), along $v\left(m ; m_{j}, u_{j}, \alpha_{2}, \ldots, \alpha_{n}+\right.$ $\left.\bar{\epsilon}_{n}\right)$ and satisfies $\beta_{n}(m,+i-1)=\delta_{i n}, 1 \leq i \leq n$. Note especially that

$$
\beta_{2}\left(m_{j}\right)=\ldots=\beta_{n}\left(m_{\jmath}\right)=0 .
$$

Simplifying our previous expression for $z_{j h}(m)$, we have

$$
\begin{aligned}
z_{j h}(m)= & \beta_{1}\left(m ; v\left(m ; m_{j}, u_{j}+\bar{h}, \alpha_{2}+\epsilon_{2}, \ldots, \alpha_{n}+\epsilon_{n}\right)\right) \\
& +\beta_{2}\left(m ; v\left(m ; m_{\jmath}, u_{j}, \alpha_{2}+\bar{\epsilon}_{2}, \ldots, \alpha_{n}+\epsilon_{n}\right)\right) \frac{\epsilon_{2}}{h} \\
& +\ldots+\beta_{n}\left(m ; v\left(m ; m_{\jmath}, u_{j}, \alpha_{2}, \ldots, \alpha_{n-1}, \alpha_{n}+\bar{\epsilon}_{n}\right)\right) \frac{\epsilon_{n}}{h} .
\end{aligned}
$$

Thus, to show $\lim _{h \rightarrow 0} z_{j h}(m)$ exists, it now suffices to show that $\lim _{h \rightarrow 0} \frac{\epsilon_{1}}{h}$ exists, for $i=2, \ldots, n$.

Now, recall that $z_{j h}\left(m_{1}\right)=\ldots=z_{j h}\left(m_{j-1}\right)=z_{j h}\left(m_{j+1}\right)=\ldots=z_{j h}\left(m_{n}\right)=0$. From our last expression for $z_{j h}(m)$, we can thus write,

$$
\begin{aligned}
- & \beta_{1}\left(m_{i} ; v\left(m ; m_{j}, u_{j}+\bar{h}, \alpha_{2}+\epsilon_{2}, \ldots, \alpha_{n}+\epsilon_{n}\right)\right) \\
= & \frac{\epsilon_{2}}{h} \beta_{2}\left(m_{i} ; v\left(m ; m_{j}, u_{j}, \alpha_{2}+\bar{\epsilon}_{2}, \ldots, \alpha_{n}+\epsilon_{n}\right)\right) \\
& \quad+\ldots+\frac{\epsilon_{n}}{h} \beta_{n}\left(m_{i} ; v\left(m ; m_{j}, u_{j}, \alpha_{2}, \ldots, \alpha_{n-1}, \alpha_{n}+\bar{\epsilon}_{n}\right)\right),
\end{aligned}
$$

for $1 \leq i \leq j-1$ and $j+1 \leq i \leq n$. This gives us a system of $n-1$ equations in the $n-1$ unknowns $\frac{c_{2}}{h}, \frac{\epsilon_{3}}{h}, \ldots, \frac{e_{n}}{h}$.

By Cramer's rule, (and suppressing the variable dependency in $v(\cdot)$ ), 


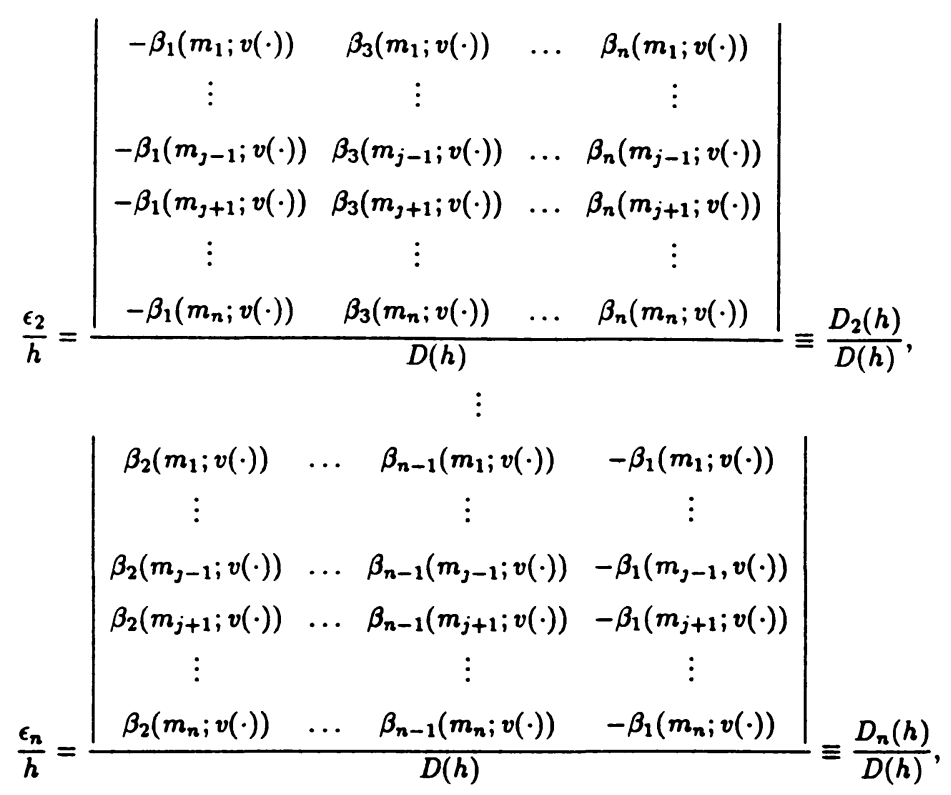

provided that

$$
0 \neq D(h) \equiv\left|\begin{array}{cccc}
\beta_{2}\left(m_{1} ; v(\cdot)\right) & \beta_{3}\left(m_{1} ; v(\cdot)\right) & \ldots & \beta_{n}\left(m_{1} ; v(\cdot)\right) \\
\vdots & \vdots & & \vdots \\
\beta_{2}\left(m_{j-1} ; v(\cdot)\right) & \beta_{3}\left(m_{j-1} ; v(\cdot)\right) & \ldots & \beta_{n}\left(m_{j-1} ; v(\cdot)\right) \\
\beta_{2}\left(m_{j+1} ; v(\cdot)\right) & \beta_{3}\left(m_{j+1} ; v(\cdot)\right) & \ldots & \beta_{n}\left(m_{j+1}, v(\cdot)\right) \\
\vdots & \vdots & & \vdots \\
\beta_{2}\left(m_{n} ; v(\cdot)\right) & \beta_{3}\left(m_{n} ; v(\cdot)\right) & \ldots & \beta_{n}\left(m_{j+1} ; v(\cdot)\right)
\end{array}\right| .
$$

To see that, for $h$ small, $D(h) \neq 0$, consider the determinant,

$$
D \equiv\left|\begin{array}{ccc}
\beta_{2}\left(m_{1} ; v\left(m ; m_{j}, u_{j}, \alpha_{2}, \ldots, \alpha_{n}\right)\right) & \ldots & \beta_{n}\left(m_{1} ; v\left(m ; m_{j}, u_{j}, \alpha_{2}, \ldots, \alpha_{n}\right)\right) \\
\vdots & & \vdots \\
\beta_{2}\left(m_{j-1} ; v\left(m ; m_{j}, u_{j}, \alpha_{2}, \ldots, \alpha_{n}\right)\right) & \ldots & \beta_{n}\left(m_{j-1} ; v\left(m ; m_{j}, u_{j}, \alpha_{2}, \ldots, \alpha_{n}\right)\right) \\
\beta_{2}\left(m_{j+1} ; v\left(m ; m_{j}, u_{j}, \alpha_{2}, \ldots, \alpha_{n}\right)\right) & \ldots & \beta_{n}\left(m_{j+1} ; v\left(m ; m_{j}, u_{j}, \alpha_{2}, \ldots, \alpha_{n}\right)\right) \\
\vdots & & \vdots \\
\beta_{2}\left(m_{n} ; v\left(m ; m_{j}, u_{j}, \alpha_{2}, \ldots, \alpha_{n}\right)\right) & \ldots & \beta_{n}\left(m_{n} ; v\left(m ; m_{j}, u_{j}, \alpha_{2}, \ldots, \alpha_{n}\right)\right)
\end{array}\right| .
$$

Now, if $D=0$, then there exists a nontrivial linear combination, for some $r_{2}, \ldots, r_{n}$, not all zero,

$$
\gamma(m) \equiv r_{2} \beta_{2}\left(m ; v\left(m ; m_{j}, u_{j}, \alpha_{2}, \ldots, \alpha_{n}\right)\right)+\ldots+r_{n} \beta_{n}\left(m ; v\left(m ; m_{j}, u_{j}, \alpha_{2}, \ldots, \alpha_{n}\right)\right),
$$

which is a solution of the variational equation, (1.2), along $v\left(m ; m_{j}, u_{j}, \alpha_{2}, \ldots, \alpha_{n}\right)$, and which also vanishes at $m=m_{1}, \ldots, m_{j-1}, m_{j+1}, \ldots, m_{n}$. It follows as before that, $\beta_{2}\left(m_{j}\right)=\ldots=\beta_{n}\left(m_{j}\right)=0$, so that $\gamma(m)$ also vanishes at $m_{j}$. Specifically, $\gamma(m)$ is a nontrivial solution of (1.2) that has $n$ zeros, which is a contradiction.

Thus, $D \neq 0$, and so by continuity, for $h$ sufficiently small, $D(h) \neq 0$. As a consequence, for every $2 \leq i \leq n$, we have that $\lim _{h \rightarrow 0} \frac{e_{h}}{h}$ exists. Say,

$$
\lim _{h \rightarrow 0} \frac{\epsilon_{i}}{h}=k_{i}, 2 \leq i \leq n
$$


As a result,

$$
\begin{aligned}
z_{j}(m) \equiv & \lim _{h \rightarrow 0} z_{j h}(m) \\
= & \beta_{1}\left(m ; v\left(m ; m_{j}, u_{j}, \alpha_{2}, \ldots, \alpha_{n}\right)\right) \\
& +k_{2} \beta_{2}\left(m ; v\left(m ; m_{j}, u_{j}, \alpha_{2}, \ldots, \alpha_{n}\right)\right) \\
& +\ldots+k_{n} \beta_{n}\left(m ; v\left(m ; m_{j}, u_{j}, \alpha_{2}, \ldots, \alpha_{n}\right)\right)
\end{aligned}
$$

exists. That is, $\frac{\partial_{u}}{\partial u_{j}}\left(m ; m_{1}, \ldots, m_{n}, u_{1}, \ldots, u_{n}\right)$ exists, and specifically, $z_{j}(m)=\frac{\partial u}{\partial u_{j}}(m)$. Moreover, since $\beta_{\imath}\left(m ; v\left(m ; m_{\jmath}, u_{\jmath}, \alpha_{2}, \ldots, \alpha_{n}\right)\right), 1 \leq i \leq n$, are all solutions of the variational equation, (1.2), along $v\left(m ; m_{j}, u_{j}, \alpha_{2}, \ldots, \alpha_{n}\right)=u\left(m ; m_{1}, \ldots, m_{n}, u_{1}, \ldots, u_{n}\right)$, we have that $z,(m)=\frac{\partial u}{\partial u_{j}}(m)$ is a solution of (1.2) along $u\left(m ; m_{1}, \ldots, m_{n}, u_{1}, \ldots, u_{n}\right)$. Also, by earlier observations,

$$
z_{j}\left(m_{i}\right)=\lim _{h \rightarrow 0} z_{j h}\left(m_{i}\right)=\delta_{i j}, 1 \leq i \leq n
$$

The proof is complete.

\section{REFERENCES}

1. HARTMAN, P. Difference equations: disconjugacy, principal solutions, Green's functions, complete monotonicity, Trans. Amer. Math. Soc. 246 (1978) 1-30.

2. AHLBRANDT, C. AND HOOKER, J. Disconjugacy criteria for second order linear difference equations, in "Proceedings, 1984 Edmonton Conference on Qualitative Properties of Differential Equations" (W. Allegretto and G. J. Butler, Eds.), pp. 15-26. University of Alberta, 1986.

3. ELOE, P. W. Difference equations and multipoint boundary value problems, Proc. Amer. Math. Soc. 86 (1982) 253-259.

4. ELOE, P. W. A boundary value problem for a system of difference equations, Nonlinear Anal. 7 (1983) 813-820.

5. HANKERSON, D. Boundary Value Problems for $n^{\text {th }}$ Order Difference Equations, Ph.D. dissertation, University of Nebraska-Lincoln, 1986.

6. HANKERSON, D. An existence and uniqueness theorem for difference equations, SIAM J. Math. Anal. 20 (1989) 1208-1217.

7. HANKERSON, D. AND PETERSON, A. A classification of the solutions of a difference equation according to their behavior at infinity, J. Math. Anal. Appl. 136 (1988) 249-266.

8. HOOKER, J. AND PATULA, W. A second-order nonlinear difference equation: oscillation and asymptotic behavior, J. Math. Anal. Appl. 91 (1983) 9-29.

9. LADAS, G., PHILOS, Ch. G. AND SFICAS, Y. G. Necessary and sufficient conditions for the oscillation of difference equations, Libertas Mathematica IX (1989).

10. PETERSON, A. Boundary value problems for an $n^{\text {th }}$ order linear difference equation SIAM J. Math. Anal. 15 (1984) 124-132.

11. PETERSON, A. Green's functions for $(k, n-k)$ boundary value problems for linear difference equations J. Math. Anal. Appl. 124 (1987) 127-138.

12. PETERSON, A. Existence and uniqueness theorems for nonlinear difference equations, $J$. Math. Anal. Appl. 125 (1987) 185-191.

13. SMITH, B. AND TAYLOR, W. Jr. Oscillatory and asymptotic behavior of certain fourth order difference equations, Rocky Mountain J. Math. 16 (1986) 403-406.

14. ELOE, P. W. Criteria for right disfocality of linear difference equations, J. Math. Anal. Appl. 120 (1986) 610-621.

15. ELOE, P. W. A comparison theorem for linear difference equations, Proc. Amer. Math. Soc. 103 (1989) 451-457.

16. ELOE, P. W. Eventual disconjugacy and right disfocality of linear difference equations, Canad. Math. Bull. 31 (1988) 362-373. 
17. PEIL, T. Criteria for Disconjugacy and Disfocality for an $n^{\text {th }}$ Order Linear Difference Equation, Ph.D. dissertation, University of Nebraska-Lincoln, 1990.

18. AGARWAL, R. P. Initial and boundary value problems for $\boldsymbol{n}^{\text {th }}$ order difference equations, Math. Slovaca 36 (1986) 39-47.

19. HENDERSON, J. Focal boundary value problems for nonlinear difference equations $I, J$. Math. Anal. Appl. 141 (1989) 559-567.

20. HENDERSON, J. Focal boundary value problems for nonlinear difference equations II, $J$. Math. Anal. Appl. 141 (1989) 568-579.

21. HENDERSON, J. Existence theorems for boundary value problems for $\boldsymbol{n}^{\text {th }}$ order nonlinear difference equations, SIAM J. Math. Anal. 20 (1989) 468-478.

22. HARTMAN, P. Ondinary Differential Equations, John Wiley and Sons, Inc., 1964.

23. HENDERSON, J. Right focal point boundary value problems for ordinary differential equations and variational equations, J. Math. Anal. Appl. 88 (1984) 363-377.

24. HENDERSON, J. Disconjugacy, disfocality, and differentiation with respect to boundary conditions, J. Math. Anal. Appl. 121 (1987) 1-9.

25. BRANTLEY, R. AND HENDERSON, J. Some analogues of a theorem of Peano for boundary value problems, Tamkang J. Math., in press. 


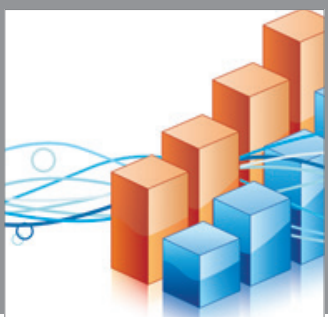

Advances in

Operations Research



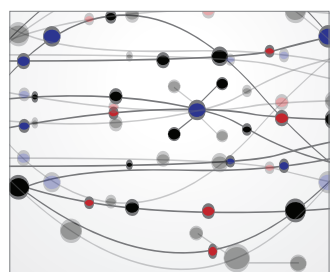

\section{The Scientific} World Journal
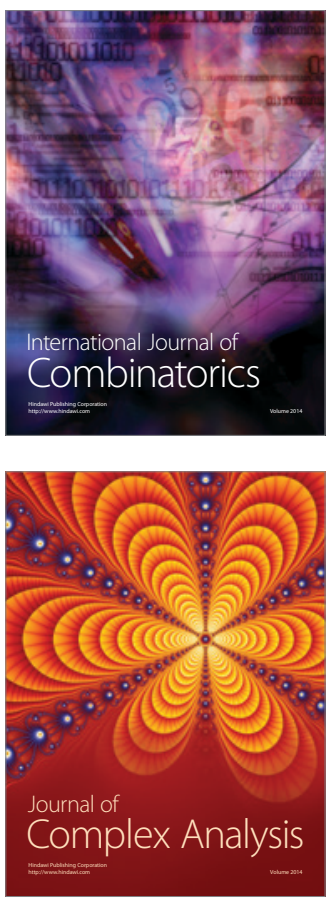

International Journal of

Mathematics and

Mathematical

Sciences


Journal of

Applied Mathematics
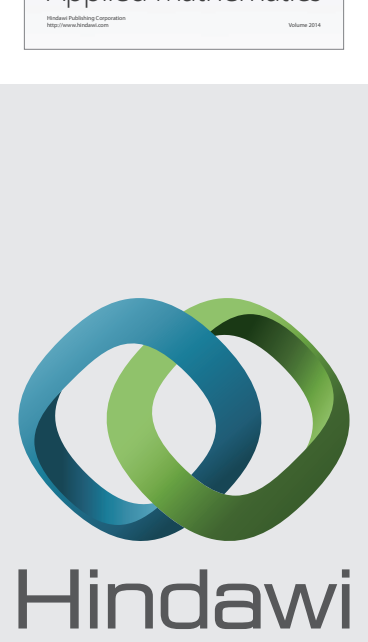

Submit your manuscripts at http://www.hindawi.com


Mathematical Problems in Engineering
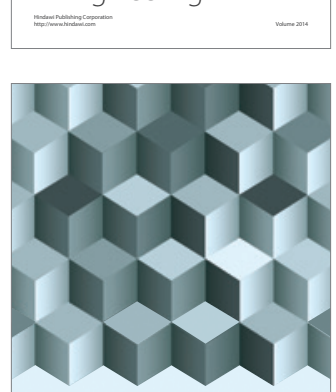

Journal of

Function Spaces




ournal of

Probability and Statistics

Promensencen
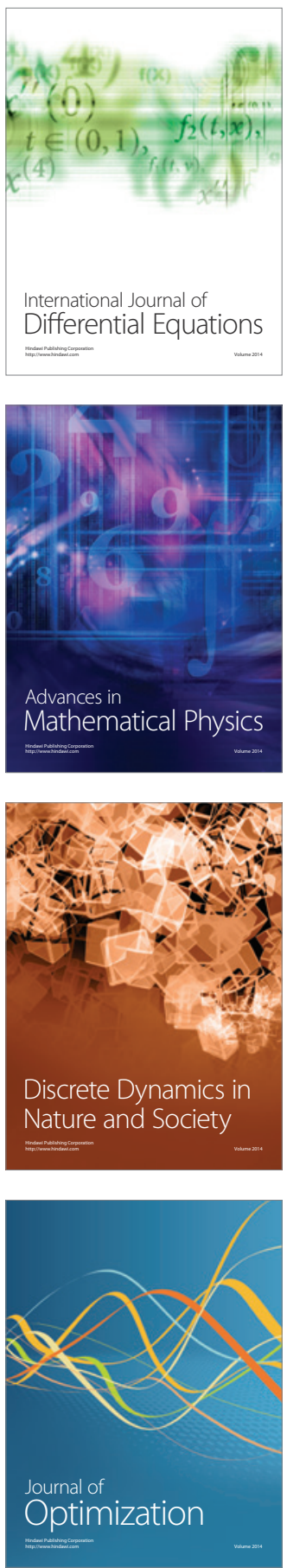